\title{
Joint Rate and Power Adaptation for Wireless Local Area Networks in Generalized Nakagami Fading Channels
}

\author{
Li-Chun Wang, Senior Member, IEEE, Wei-Cheng Liu, Member, IEEE, \\ Anderson Chen, Member, IEEE, and Kuang-Nan Yen
}

\begin{abstract}
In this paper, a channel-driven rate and power adaptation (CDRPA) algorithm is proposed for wireless local area networks (WLANs) in generalized Nakagami fading channels. Through the channel information of the first packet, the CDRPA algorithm can lower the complexity of comparing various rates and power levels. In the case of the IEEE 802.11 WLAN, the computation complexity of the CDRPA algorithms is reduced by more than $94 \%$ compared with the complete-search link adaptation. A physical and/or medium access control (MAC) cross-layer analytical model is also developed to evaluate the goodput and energy efficiency of the carrier sense multiple access with a collision avoidance MAC protocol in Nakagami fading channels. Our numerical results show that the CDRPA algorithm is not only more energy efficient in the Nakagami fading channel but can also achieve comparable and even higher throughput compared with the complete-search link adaptation approach.
\end{abstract}

Index Terms-Channel-driven rate and power adaptation (CDRPA), IEEE 802.11, medium access control (MAC), Nakagami fading channels, wireless local area networks (WLANs).

\section{INTRODUCTION}

$\mathbf{I}$ MPROVING energy efficiency and enhancing throughput are two important objectives for wireless local area networks (WLANs). To prolong a battery's life time, the IEEE 802.11h standard body has specified a report mechanism to dynamically adjust the transmitted power in WLAN [1]. It is well known that the IEEE $802.11 \mathrm{a} / \mathrm{g}$ WLAN can improve throughput ${ }^{1}$ by appropriately changing eight modulation and coding schemes (MCSs) according to the channel quality, as shown in Table I. Thus, an interesting but challenging issue arises: How can the MCS and transmitted power be jointly adapted to si-

Manuscript received June 7, 2006; revised July 4, 2007, November 20, 2007 , April 21, 2008, and June 9, 2008. First published July 16, 2008; current version published March 17, 2009. This work was supported by in part by the Ministry of Education under the Aiming for the Top University and Elite Research Center Development (ATU) Plan and by the National Science Council, Taiwan, under Contract NSC96-2628-E-009-004-MY3. The review of this paper was coordinated by Dr. W. Zhuang.

L.-C. Wang and W.-C. Liu are with the Department of Communication Engineering, National Chiao Tung University, Hsinchu 300, Taiwan (e-mail: lichun@mail.nctu.edu.tw; wcliu@faculty.nctu.edu.tw).

A. Chen is with national service (e-mail: mingbing.cm87g@nctu.edu.tw).

K.-N. Yen is with TrendChip Technologies Corp., Hsinchu 300, Taiwan (e-mail: kuangnan.cm92g@nctu.edu.tw).

Color versions of one or more of the figures in this paper are available online at http://ieeexplore.ieee.org.

Digital Object Identifier 10.1109/TVT.2008.928641

${ }^{1}$ Hereafter, throughput and goodput, which is defined as the successfully delivered data payload, excluding the overhead, will be used interchangeably.
TABLE I

Eight Modulation And Coding Schemes For the Associated DATA RATES IN THE IEEE $802.11 \mathrm{a} / \mathrm{g}$ WLAN

\begin{tabular}{|c|c|c|c|c|}
\hline Mode $M$ & Data Rate & Modulation & Coding rate & $\mathrm{BpS}(M)^{*}$ \\
\hline \hline 1 & $6 \mathrm{Mbps}$ & BPSK & $1 / 2$ & 3 \\
\hline 2 & $9 \mathrm{Mbps}$ & BPSK & $3 / 4$ & 4.5 \\
\hline 3 & $12 \mathrm{Mbps}$ & QPSK & $1 / 2$ & 6 \\
\hline 4 & $18 \mathrm{Mbps}$ & QPSK & $3 / 4$ & 9 \\
\hline 5 & $24 \mathrm{Mbps}$ & 16-QAM & $1 / 2$ & 12 \\
\hline 6 & $36 \mathrm{Mbps}$ & 16-QAM & $3 / 4$ & 18 \\
\hline 7 & $48 \mathrm{Mbps}$ & 64-QAM & $2 / 3$ & 24 \\
\hline 8 & $54 \mathrm{Mbps}$ & 64-QAM & $3 / 4$ & 27 \\
\hline
\end{tabular}

*BpS: Bytes per OFDM Symbol

multaneously enhance throughput and energy efficiency? The main challenge of joint rate and power adaptation lies in the fact that energy efficiency and throughput are, indeed, two contradictory objectives. More efficient modulation and fewer redundant coding bits can achieve higher throughput. However, a more efficient MCS also requires higher transmitted power to maintain the bit error rate (BER) performance.

One of the keys to designing effective link adaptation mechanisms is to incorporate channel effects of the physical (PHY) layer into the decision-making process of the medium access control (MAC) layer. Fast varying multipath fading significantly affects the selection of MCS. In particular, when the punctured bits is used, such as the $3 / 4$ coding rate with respect to 9,18 , and $36 \mathrm{Mb} / \mathrm{s}$ in the IEEE $802.11 \mathrm{a} / \mathrm{g}$ WLAN, the performance of MCS significantly degrades if an inappropriate MCS is selected. Another key factor is to design a retransmission strategy for the joint rate and power adaptation when the channel information is unavailable. In WLAN, through the received acknowledgement (ACK) frame, the source station can acquire the channel condition. If the previous data frame is collided or the received ACK frame corrupted, the source station cannot effectively adjust the transmitted rate and power according to the channel-state information.

The objective of this paper is to investigate how the IEEE 802.11 WLAN can decide on an appropriate set of MCS and transmitted power to optimize the tradeoff between energy efficiency and goodput in generalized Nakagami fading channels. The Rayleigh fading is the special case of the Nakagami fading channel. In this paper, we develop two channel-driven rate and power adaptation (CDRPA) algorithms based on energy efficiency and goodput aspects, respectively. On one hand, the power-first CDRPA scheme first selects the transmitted power 
TABLE II

TransmitTED POWER LEVELS FOR THE IEEE 802.11 WLAN AT THE 5-GHz ISM BAND

\begin{tabular}{|c|c|}
\hline Frequency Band & $\begin{array}{c}\text { Maximum Transmitted Power } \\
\text { with 6 dBi Antenna Gain }\end{array}$ \\
\hline \hline $5.150-5.250 \mathrm{GHz}$ & $40 \mathrm{~mW}(16 \mathrm{dBm})$ \\
\hline $5.250-5.350 \mathrm{GHz}$ & $200 \mathrm{~mW}(23 \mathrm{dBm})$ \\
\hline $5.725-5.825 \mathrm{GHz}$ & $800 \mathrm{~mW}(29 \mathrm{dBm})$ \\
\hline
\end{tabular}

to improve energy efficiency and then decides on an appropriate data rate for transmissions. On the other hand, the rate-first CDRPA scheme places higher priority on goodput enhancement than energy savings. Determining the optimal combination of MCS and transmitted power out of many choices is not an easy task, as shown in Tables I and II. Exploiting the channel information, the CDRPA algorithms can reduce the number of comparisons among all the possible combinations of MCS and power levels. In addition, a step-down retransmission strategy is also designed for a station to retransmit the packet. The proposed strategy adjusts the transmitted rate and power according to the selection in the previous transmission attempt. A station does not have prior knowledge of channel quality in the case of frame retransmission; thus, the designed strategy is quite feasible from the implementation aspect.

Another important question is as follows: What are the performance differences between CDRPA algorithms and the energy-optimal/goodput-optimal link adaptation schemes that exclusively search all the possible combinations of MCS and transmitted power levels? We develop a PHY/MAC cross-layer analytical model to evaluate the goodput and energy efficiency of the carrier sense multiple access with collision avoidance (CSMA/CA) MAC protocol in the generalized Nakagami fading channel. Based on the numerical results, the power-first CDRPA algorithm maintains comparable goodput performance as the throughput-optimal link adaptation scheme [2] in the generalized Nakagami fading channel. In the additive white Gaussian noise (AWGN) channel, the power-first scheme can not only achieve the same energy efficiency and throughput as the energy-optimal MiSer algorithm [3] but can also provide higher throughput in the Nakagami fading channel. Compared with the power-first CDRPA and MiSer algorithms, the rate-first CDRPA algorithm can support higher throughput at the highSNR region in the AWGN channel but yields lower throughput at the low-SNR region. In general, the rate-first CDRPA can achieve higher throughput in the Nakagami fading channel at the cost of lower energy efficiency. On the other hand, the power-first CDRPA can achieve the same energy efficiency performance as the energy-optimal MiSer algorithm and maintain high throughput.

The rest of this paper is organized as follows. Section II discusses the related work. In Section III, we introduce the Nakagami fading channel model and the specifications of the IEEE 802.11a/g WLAN in the PHY and MAC layers. The CDRPA mechanisms are presented in Section IV. In Section V, we develop a PHY/MAC cross-layer analytical method, which can evaluate the goodput and energy efficiency of the CSMA/CA MAC protocol in the Nakagami fading channel. Section VI shows the numerical results. Section VII gives the concluding remarks.

\section{RELATED WORK}

In the literature, the rate adaptation and power control mechanisms for the IEEE 802.11 WLAN were mainly designed to separately improve goodput and energy efficiency but not both.

First, let us discuss some adaptation schemes that aim at improving the energy efficiency of WLANs.

- In [4] and [5], the proposed algorithms dynamically turn off unnecessary functions to save energy consumption.

- In [6], a simple path-loss-based power control scheme was proposed for the IEEE 802.11a/g WLAN. According to the difference of the transmitted and received power in the Request-to-Send (RTS) frame, the estimated path loss is inserted into the Clear-to-Send (CTS) frame. Upon receiving the CTS frame, the source station will accordingly adjust the transmitted power based on the estimated path loss.

- In [3], Qiao et al. proposed a minimum-energy transmission strategy called the MiSer algorithm in the AWGN channel. This algorithm can maximize the ratio of the successfully received bits over the consumed energy. Because of a complete search among all the combinations of the transmitted power levels and the MCS, the MiSer algorithm can be viewed as the optimal energy-efficiency algorithm in the AWGN channel.

Next, let us discuss the existing rate-adaptation schemes, with the objective of enhancing the goodput performance for the WLAN.

- One well-known rate adaptation scheme was the auto rate fallback (ARF) algorithm [7]. The basic idea of ARF was to count the number of successfully received and missing ACK frames. On one hand, after failing to consecutively receive two ACK frames, the transmitter lowers the data rate in the next retransmission. On the other hand, if ten ACK frames are successfully received, the transmitter changes to the next higher data rate. One improved version with adaptively successful and failure transmission threshold was proposed in [8]. In [9], another rate-adaptation scheme based on the long- and short-term statistics of successful and failed transmitted frames was proposed. These algorithms took a long period of time to count the number of ACK frames; thus, the ARF algorithm and the statistics-based rate adaptation schemes may not react fast enough to follow the fluctuations of the wireless channel.

- To expedite the rate adaptation process, some fast-rate adaptation algorithms [2], [10]-[13] were proposed without counting the number of received ACK frames.

- In [10] and [11], the algorithms mandated the RTS/CTS handshaking to acquire the channel quality. The source station adapts the transmitted rate by the path loss or SNR information that was inserted into the CTS frame. Obviously, both works required changing the IEEE 802.11 Standard; thus, it is not practical to deploy them in existing WLANs.

- In [2], the proper MCS $M$ is selected based on the payload length $l$, the received signal quality of the ACK frame $s$, and the retry count $n$. Exhaustively searching all the combinations of $(M, l, s, n)$, the source station selects 
the combination with the highest goodput. This approach resulted in the "optimum" goodput performance in the AWGN channel. However, the complexity of searching all the combinations of $(M, l, s, n)$ can be a concern in terms of power saving. Furthermore, the optimal choice of $(M, l, s, n)$ in the AWGN channel may not be suitable in the multipath fading channel.

- In [12] and [13], the authors suggested a reduced PHYmode table that removes some unnecessary modes in the Nakagami fading channels for rate-adaptation to reduce the computation complexity. Even in the AWGN channel, the reduced mode rate-adaptation schemes in [12] and [13] can deliver comparable goodput performance with the complete searching rate-adaptation scheme in [2].

To our knowledge, a joint adaptation rate and power mechanism that aims at simultaneously improving the energy efficiency and goodput performance is rare in the literature. The key issue in the joint rate and power adaptation is to design a fast feasible rule to simultaneously determine the transmitted power and rate. In the literature, most works adapt the transmitted power and rate according to either a long-term observation of the successful and failure frame transmissions or the channelstate information prior to each transmission attempt. A wireless channel varies by time; therefore, the works based on the time-costly statistical method are not effective for the joint rate and power adaptation in WLAN. Furthermore, unlike the cellular network, the source station in IEEE 802.11 WLANs may not always have channel-state information, particularly when it retransmits frames. Therefore, a joint rate and power adaptation algorithm that requires the channel-state information in advance of each retransmission is still an issue in IEEE 802.11 WLANs. Thus, in this paper, the proposed CDRPA algorithms aim at overcoming the two aforementioned issues, with the objective of optimizing the tradeoff between energy efficiency and goodput in WLAN.

\section{SYSTEM MODEL}

\section{A. IEEE 802.11a/g PHY and MAC}

The PHY layer of the IEEE 802.11a/g WLAN provides eight different data rates that range from 6 to $54 \mathrm{Mb} / \mathrm{s}$, as shown in Table I. The maximum transmitted power levels at $5-\mathrm{GHz}$ industrial, scientific, and medical (ISM) bands are listed in Table II [14], [15].

In the MAC layer, the distributed coordination function (DCF) based on the CSMA/CA MAC protocol is, accordingly, applied to grant access of the common medium among users [16]. Fig. 1 demonstrates three frame transmission scenarios in the basic mode of DCF.

1) Successful data and ACK frames transmissions. The source station successfully sends out a data frame after the duration of the random backoff and the distributed interframe space (DIFS). Next, the source station waits for the duration of a short interframe space (SIFS) and then successfully receives an ACK control frame from its destination.

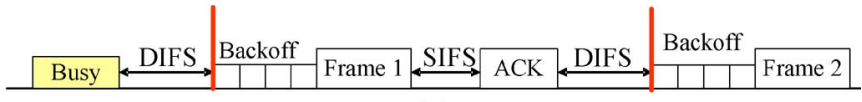

(a)

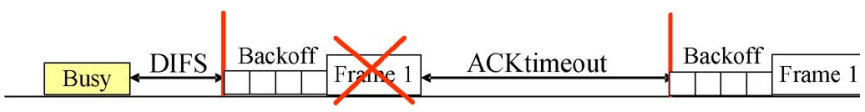

(b)

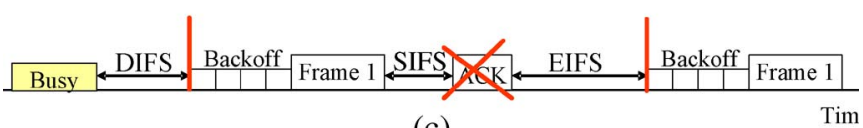

(c)

Fig. 1. Three scenarios of frame transmission according to the CSMA/CA MAC protocol. (a) Successful frame transmission. (b) Erroneous data frame transmission. (c) Erroneous ACK frame transmission.

2) Erroneous data frame transmission. If the ACK frame is not received within the duration of ACK timeout after data frame 1 is sent out, the source station will retransmit the data frame.

3) Erroneous ACK frame transmission. If the ACK frame is received but corrupted, the source station waits for the duration of the extended interframe space (EIFS) and retransmits data frame 1 . Note that EIFS is longer than DIFS and SIFS.

\section{B. Generalized Nakagami Fading Channels}

Consider a generalized Nakagami fading channel [17], of which the probability density function (pdf) of the signal amplitude is given by

$$
p(\alpha)=\frac{2 m^{m} \alpha^{2 m-1}}{\Omega^{m} \Gamma(m)} \exp \left(-\frac{m \alpha^{2}}{\Omega}\right)
$$

where $\alpha, m$, and $\Omega$ are the faded amplitude, the Nakagami fading parameter, and the local mean power, respectively, and $\Gamma(m)$ is the Gamma function. The Nakagami channel model becomes the Rayleigh fading channel for $m=1$, but it becomes the nonfaded channel as $m \rightarrow \infty$.

\section{Channel Transition Probability Matrix}

In principle, a wireless fading channel can be modeled by many states according to the channel quality. A well-known simple model is the Gilbert channel model, which is only classified into "on/off' two states. In [12], a wireless fading channel is divided into eight states $s_{j}$, each of which is associated with the required $E_{b} / N_{0}$ threshold $\eta_{j}$ for data rates $j=1 \sim 8$ in the IEEE $802.11 \mathrm{a} / \mathrm{g}$ WLAN. In particular, the state variable $s_{j}$ is defined as the event that the received $E_{b} / N_{0} \in\left[\eta_{j-1}, \eta_{j}\right)$, which the $E_{b} / N_{0}$ threshold $\left(\eta_{j}\right)$ can obtain from the offline PHY-layer simulation.

Denote $x_{i}$ as the received $E_{b} / N_{0}$ at the $i$ th transmission. Then, the channel transition probability $p_{j k}$ from states $s_{j}$ to $s_{k}$ can be expressed as

$$
\begin{aligned}
p_{j k} & =P\left(x_{i+1} \in\left(\eta_{k-1}, \eta_{k}\right) \mid x_{i} \in\left(\eta_{j-1}, \eta_{j}\right)\right) \\
& =\frac{\int_{\eta_{k-1}}^{\eta_{k}} \int_{\eta_{j-1}}^{\eta_{j}} p\left(\alpha_{i}, \alpha_{i+1}\right) d \alpha_{i} d \alpha_{i+1}}{\int_{0}^{\infty} \int_{\eta_{j-1}}^{\eta_{j}} p\left(\alpha_{i}, \alpha_{i+1}\right) d \alpha_{i} d \alpha_{i+1}} .
\end{aligned}
$$


In a Nakagami fading channel, the joint pdf $p\left(\alpha_{t-\tau}, \alpha_{t}\right)$ of two channel estimations $\alpha_{t-\tau}$ and $\alpha_{t}$ at time instant $(t-\tau)$ and $t$ can be computed by

$$
\begin{aligned}
p\left(\alpha_{t-\tau}, \alpha_{t}\right)= & \frac{4\left(\alpha_{t-\tau} \alpha_{t}\right)^{m}}{\left(1-\rho_{2}\right) \Gamma(m) \rho_{2}^{(m-1) / 2}}\left(\frac{m}{\Omega}\right)^{m+1} \\
& \times I_{m-1}\left(\frac{2 m \sqrt{\rho_{2}} \alpha_{t-\tau} \alpha_{t}}{\left(1-\rho_{2}\right) \Omega}\right) \\
& \times \exp \left(-\frac{m\left(\alpha_{t-\tau}^{2}+\alpha_{t}^{2}\right)}{\left(1-\rho_{2}\right) \Omega}\right)
\end{aligned}
$$

where $I_{m-1}(\cdot)$ is the $(m-1)$ th-order modified Bessel function of the first kind, $E\left[\alpha_{t-\tau}^{2}\right]=E\left[\alpha_{t}^{2}\right]=\Omega$ is the average local mean power, and $\rho_{2}$ is the power correlation coefficient between fading power $\alpha_{t}^{2}$ and $\alpha_{t-\tau}^{2}$ and is a function of $\tau$. Referring to [18], a plausible model of $\rho_{2}(\tau)$ is given by

$$
\rho_{2}(\tau)=\left|\frac{I_{0}\left(\sqrt{\kappa^{2}-\left(2 \pi f_{D} \tau\right)^{2}+j 4 \pi \kappa f_{D} \tau \cos \mu}\right)}{I_{0}(\kappa)}\right|^{2}
$$

where $f_{D}$ is the maximum Doppler frequency (in hertz), $\mu$ is the mean direction of the angle of arrival (AOA), and $\kappa$ is the beamwidth parameter.

\section{Rate And Power Adaptation Mechanism}

In this section, we describe two proposed joint rate and power adaptation algorithms: 1) the power-first algorithm and 2) the rate-first CDRPA algorithm. In addition, we also illustrate the designed step-down retransmission strategy for packet retransmission.

\section{A. CDRPA Algorithm}

The basic idea of the CDRPA algorithm is to adjust the transmitted rate and power according to the received $E_{b} / N_{0}$ of the previous ACK frame. The packet transmissions in WLAN are in the same frequency band. It is assumed that both the link channels have similar fading characteristics, because the forward and reverse links in WLAN are separated by time. The received $E_{b} / N_{0}$ of the previously received ACK frame can be assumed to be an indicative channel-state information for the next packet transmission. In Fig. 2, the procedures of the CDRPA algorithm are elaborated as follows.

1) ACK frame reception. The source station first checks whether the previous ACK frame is successfully received.

a) If no ACK frame in the previous packet transmission is successfully received, the station first chooses the lowest (i.e., the most robust) PHY mode and the highest transmitted power for the next frame transmission.

b) If an ACK frame is successfully received, the transmitted rate and power is adjusted based on the received $E_{b} / N_{0}$ of the ACK frame for the next frame transmission.

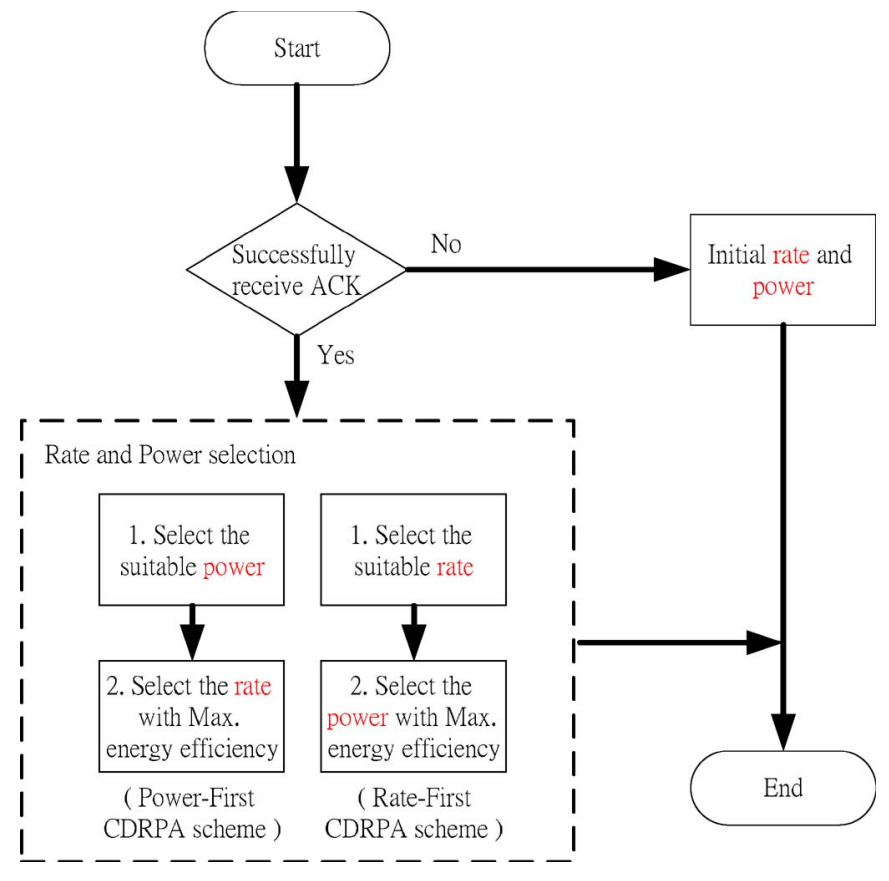

Fig. 2. Flowchart of the CDRPA algorithm.

2) Transmitted power selection. Calculate the backoff power level only for the efficient PHY modes according to the received $E_{b} / N_{0}$ of the previous ACK frame.

3) Transmitted rate selection. Select the rate based on either the most energy efficient or the highest goodput by the predetermined tables from the offline simulation and analysis.

Transmitted power selection. To decide on the transmitted power, we use the previously received ACK frame to evaluate the available link margin in the current channel. Let $E_{b} / N_{0_{r x}}$ and $\eta_{r e q}$ be the received $E_{b} / N_{0}$ of the ACK frame and the required $E_{b} / N_{0}$ threshold of the selected transmitted rate, respectively. Then, we can define the available link margin $L_{\text {margin }}$ as

$$
L_{\text {margin }}=E_{b} / N_{0_{r x}}-\eta_{r e q} .
$$

The ACK frame is always transmitted by the maximum power level $P_{t(\max )}$; thus, the available link margin $L_{\text {margin }}$ in (5) can be viewed as the amount of power that can be reduced without dropping frames. When $L_{\text {margin }}>0$, the channel condition is better than the value required by the selected PHY mode; thus, the transmitted power level can be reduced by the value of $L_{\text {margin }}$ and satisfy the packet error rate (PER) requirement. Therefore, the source station can adjust the transmitted power for the next transmission by

$$
P_{t}=\left\lceil P_{t(\max )}-L_{\operatorname{margin}}\right\rceil
$$

Transmitted rate selection. The CDRPA algorithm adopts the concept of PHY-mode reduction to select the transmitted rate based on the requirements for the lowest energy consumption or the highest goodput performance [12], [13]. The reduced-mode concept in the IEEE 802.11 WLAN removes 


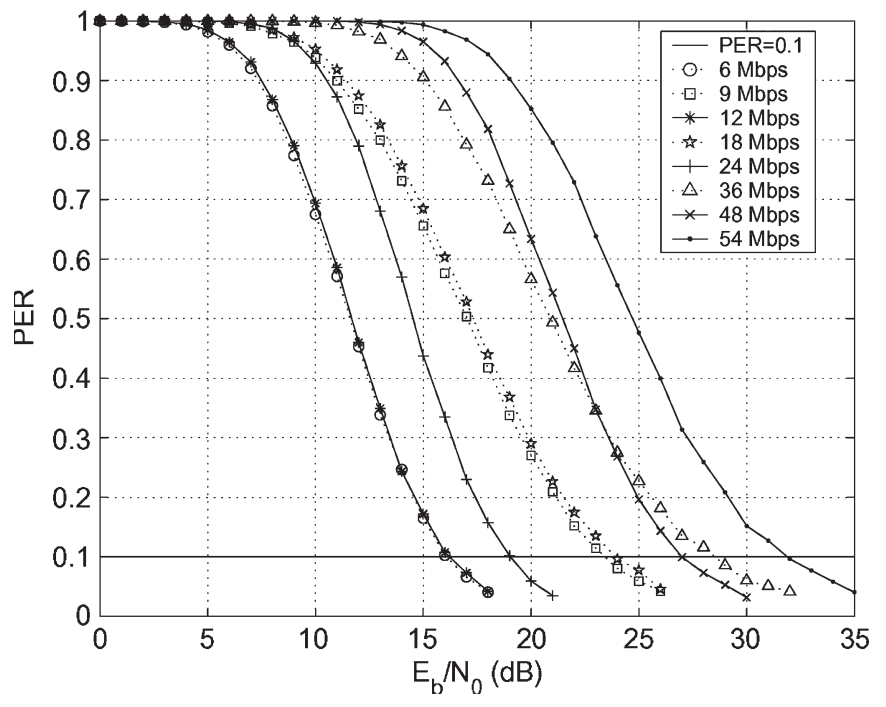

Fig. 3. Packet error rate versus $E_{b} / N_{0}$ using the PHY-layer transmission parameters of the IEEE $802.11 \mathrm{a} / \mathrm{g}$ WLAN in the Rayleigh fading channel with a payload length of $1500 \mathrm{~B}$.

TABLE III

PERFormance ReQuirement FOR THE IEEE 802.11a/g WLAN IN THE NAKAGAMI FADING CHANNEL With SHAPING FACTORS $m=1$ AND $m=5$. (a) THE REQUIRED $E_{b} / N_{0}(\eta)$ TO SUPPORT EIGHT CORRESPONDING DATA RATES WITH PER $=0.1$ AND 1500-B PAYLOAD LENGTH B y A TRANSMitTED POWER OF $30 \mathrm{dBm}$. (b) CONDENSED Channel STATES For $\mathrm{i}=1$ TO $\mathrm{i}=4$

\begin{tabular}{|c|c|c|c|c|}
\hline Data Rate $(\mathrm{Mbps})$ & 6 & 9 & 12 & 18 \\
\hline$\eta$ for $m=1$ & 16.25 & 23.80 & $\underline{16.33}$ & 24.20 \\
\hline$\eta$ for $m=5$ & 7.86 & 14.87 & $\underline{7.89}$ & 14.98 \\
\hline \hline Data Rate $(\mathrm{Mbps})$ & 24 & 36 & 48 & 54 \\
\hline$\eta$ for $m=1$ & $\underline{19.11}$ & 28.40 & $\underline{26.90}$ & $\underline{31.88}$ \\
\hline$\eta$ for $m=5$ & $\underline{10.62}$ & 18.90 & $\underline{17.78}$ & $\underline{22.56}$ \\
\hline
\end{tabular}

(a)

\begin{tabular}{|c|c|c|}
\hline channel state & $s_{1}$ & $s_{2}$ \\
\hline Data Rate $($ Mbps) & 12 & 24 \\
\hline$E_{b} / N_{0}$ range for $m=1$ & {$[0,19.11)$} & {$[19.11,26.90)$} \\
\hline$E_{b} / N_{0}$ range for $m=5$ & {$[0,10.62)$} & {$[10.62,17.78)$} \\
\hline \hline channel state & $s_{3}$ & $s_{4}$ \\
\hline Data Rate $($ Mbps) & 48 & 54 \\
\hline$E_{b} / N_{0}$ range for $m=1$ & {$[26.90,31.88)$} & {$[31.88, \infty)$} \\
\hline$E_{b} / N_{0}$ range for $m=5$ & {$[17.78,22.56)$} & {$[22.56, \infty)$} \\
\hline
\end{tabular}

(b)

inefficient PHY modes to improve goodput performance in the Nakagami fading channel while reducing the complexity. Take the IEEE $802.11 \mathrm{a} / \mathrm{g}$ WLAN as an example. Fig. 3 shows the PER against $E_{b} / N_{0}$ with 1500 -B packets for eight different data rates in the Rayleigh fading channel. For PER $=0.1$, only four PHY modes with respect to $12,24,48$, and $54 \mathrm{Mb} / \mathrm{s}$ need to be considered, as shown in Table III(a). The other modes almost have similar performance compared with the selected ones. With the $E_{b} / N_{0}$ thresholds $\eta_{i}$ for the four efficient modes $(i=1 \sim 4)$, Table III(b) lists the four channel states and the corresponding $E_{b} / N_{0}$ ranges for the IEEE 802.11 WLAN in the Rayleigh and Nakagami fading channels, with $m=5$. Therefore, although the inefficient PHY modes are removed, the rate adaptation that uses the reduced mode table still approaches the goodput performance of the complete mode.
Next, we respectively discuss the "power-first" and "ratefirst" CDRPA schemes, which achieve two different goals: maximal energy efficiency and goodput performance.

\section{B. Power-First CDRPA Scheme}

The objective of the power-first CDRPA scheme is to maximize the energy efficiency of frame transmissions. Referring to Fig. 2, the rate- and power-selection procedures of the powerfirst CDRPA scheme are described as follows.

1) After successfully receiving the $\mathrm{ACK}$ frame, determine the transmitted power for the feasible PHY modes by substituting the received $E_{b} / N_{0}$ of the ACK frame into (5) and (6).

2) Look up the energy consumption of the rates and power levels in step 1 from an established table by offline analysis.

3) Select the transmitted rate with the maximum energy efficiency for the next frame transmission.

Assume that the maximum transmitted power $p_{t(\max )}=$ $30 \mathrm{dBm}$ and that the received $E_{b} / N_{0}$ of the ACK frame is $27 \mathrm{~dB}$. In step 1, the two feasible transmitted rates and power levels are $(24 \mathrm{Mb} / \mathrm{s}, 23 \mathrm{dBm})$ and $(48 \mathrm{Mb} / \mathrm{s}, 30 \mathrm{dBm})$. Next, compare the energy consumption of the selected rates and power levels, i.e., 478.5-720.4 J. Because of lower energy consumption, the use of $24 \mathrm{Mb} / \mathrm{s}$ and $23 \mathrm{dBm}$ is chosen in the next frame transmission. Therefore, one can expect that the power-first CDRPA can maximize the energy efficiency.

\section{Rate-First CDRPA Scheme}

Unlike the power-first CDRPA scheme, the rate-first CDRPA scheme aims at maximizing the goodput performance for the frame transmissions. Referring to Fig. 2, the procedures of the rate-first CDRPA scheme are described as follows.

1) Select an appropriate PHY mode from the reduced mode table, with which the $E_{b} / N_{0}$ threshold is close but is lower than the received $E_{b} / N_{0}$ of the previous ACK frame.

2) Based on the transmitted power selection method, compute the transmitted power level by (5) and (6).

3) Use the rate and power levels in steps 1 and 2 for the next frame transmission.

For instance, when $p_{t_{\text {max })}}=30 \mathrm{dBm}$ and the received $E_{b} / N_{0}$ of the ACK frame is $28 \mathrm{~dB}$, the highest feasible transmitted rate is $48 \mathrm{Mb} / \mathrm{s}$. Next, the transmitted power level is calculated based on the link budget by (6). According to Table III, the transmitted power level is $29 \mathrm{dBm}$ due to the link budget $L_{\text {margin }}=1 \mathrm{~dB}$. Based on the above descriptions, as the received $E_{b} / N_{0}$ increases, the rate-first CDRPA scheme raises the transmitted rate instead of decreasing the transmitted power. Thus, one can expect that the rate-first CDRPA can use the highest transmitted rate at the given channel condition. The goodput performance of the rate-first CDRPA is the same as the CDRA algorithm [13], whereas the energy of the rate-first CDRPA is more efficient than the CDRA algorithm due to the additional power adaptation mechanism. 
TABLE IV

ILLUSTRATION OF THE STEP-DOWN RETRANSMISSION STRATEGY

\begin{tabular}{|c|c|c|c|c|c|c|c|}
\hline Transmission attempt & 1 & 2 & 3 & 4 & 5 & 6 & 7 \\
\hline Transmitted rate (Mbps) & 48 & 48 & 24 & 12 & 12 & 12 & 12 \\
\hline Transmitted power level $(\mathrm{dBm})$ & 26 & 30 & 30 & 30 & 30 & 30 & 30 \\
\hline
\end{tabular}

\section{Step-Down Retransmission Strategy}

In addition to the two proposed CDRPA schemes, a new stepdown retransmission strategy is designed to retransmit packets. One of the key issues in the CDRPA algorithm and other previous works is that the source station requires channel-state information from the previously received ACK frame before any packet transmission. If the ACK frame is corrupted, or the previous transmission times out, the station cannot adjust the transmitted rate and power due to the absence of the channelstate information.

The basic idea of the step-down retransmission strategy is to incorporate the ARF algorithm [7] into the newly proposed CDRPA algorithm. In the newly designed retransmission strategy, a station adjusts the transmitted rate and power based on the previously selected set and the number of transmission attempts. First, the source station determines the transmitted rate and power level based on the proposed rate-first or power-first CDRPA algorithm. When the first transmission fails, the station increases the transmitted power to the highest level $P_{t(\max )}$ but still holds the transmitted rate. Suppose that the frame transmission fails again. Then, the station lowers the transmitted rate step by step until the lowest mode in the reduced PHY-mode table. For example, when $P_{t(\max )}=30 \mathrm{dBm}$, the maximum number of transmission attempts for a packet $n_{\max }=7$, and the rate and power are $48 \mathrm{Mb} / \mathrm{s}$ and $26 \mathrm{dBm}$ for the first frame transmission, the transmitted rates, and power levels at the succeeding transmission attempts, as listed in Table IV. Apparently, the joint CDRPA algorithm with the step-down retransmission strategy not only can adapt to the fast varying wireless channel but also require no channel information when a station retransmits the packet. Thus, it is feasible in terms of implementation.

\section{Performance Analysis}

In this section, we develop a PHY/MAC cross-layer analytical model to evaluate the goodput performance and energy efficiency for the joint rate and power adaptation algorithms in the Nakagami fading channel.

\section{A. Goodput}

Effective goodput $\mathcal{G}$ is defined as the number of successfully delivered information bits per second. Denote $E\left[l_{D}\right]$ as the average delivered information bits of a data packet. Denote $E\left[t_{T}\right]$ as the average transmission time that is required by a station that successfully transmits a data packet. Then, the goodput $\mathcal{G}$ can be written as

$$
\mathcal{G}\left(l, s_{i}, M_{i}\right)=\frac{E\left[l_{D}\right]\left(l, s_{i}, M_{i}\right)}{E\left[t_{T}\right]\left(l, s_{i}, M_{i}\right)}
$$

where $\left(l, s_{i}, M_{i}\right)$ represent the data payload length, the received $E_{b} / N_{0}$, and the transmitted PHY mode at the $i$ th transmission attempt, respectively. Given the received $E_{b} / N_{0}$ of the previous ACK frame $\gamma$, the transmitted PHY modes at all transmission attempts based on the CDRPA algorithm and the step-down retransmission strategy can be expressed as $\left\{M_{1}(\gamma), M_{2}, \ldots, M_{n_{\max }}\right\}$.

The wireless channel varies by time; thus, the data packet transmission may not always be successful due to the limited number of transmission attempts. Given $p_{s}\left(l, s_{i}, M_{i}\right)$, i.e., the probability that a data packet is successfully transmitted at the $i$ th attempt, the average delivered information bits $E\left[l_{D}\right]$ can be written as

$$
\begin{array}{r}
E\left[l_{D}\right]\left(l, s_{i}, M_{i}\right)=p_{s}\left(l, s_{i}, M_{i}\right) \cdot l+\left[1-p_{s}\left(l, s_{i}, M_{i}\right)\right] \\
\times \sum_{k=1}^{N} p_{j k} \cdot E\left[l_{D}\right]\left(l, s_{i+1}, M_{i+1}\right)
\end{array}
$$

where $N$ is the number of channel states, the transition probability $p_{j k}$ represents the channel condition change from state $j$ to state $k$ at the $i$ th and $(i+1)$ th transmission attempts, as defined in (2). Furthermore, let $p_{e, d a t a}\left(l, s_{i}, M_{i}\right)$ and $p_{e, a c k}\left(s_{i}\right)$ be the error probabilities of data and ACK frames, respectively. Then, the success probability of a frame transmission $p_{s}\left(l, s_{i}, M_{i}\right)$ in (8) can be computed by

$$
p_{s}\left(l, s_{i}, M_{i}\right)=\left[1-p_{e, \text { data }}\left(l, s_{i}, M_{i}\right)\right] \cdot\left[1-p_{e, a c k}\left(s_{i}\right)\right] .
$$

The detailed expressions of $p_{e, \text { data }}\left(l, s_{i}, M_{i}\right)$ and $p_{e, a c k}\left(s_{i}\right)$ can be found in [12].

Next, we compute the average transmission time $E\left[t_{T}\right]$ in (7). According to the definition, $E\left[t_{T}\right]\left(l, s_{i}, M_{i}\right)$ accounts for the total time duration starting from the moment when a source station sends a packet until it successfully receives the ACK frame from its destination station or reaches the number of maximum transmission attempts. Recall that a data frame transmission, based on the legacy CSMA/CA MAC protocol, has three different scenarios, as shown in Fig. 1. Denote $T_{a}, T_{b}$ and $T_{c}$ as the duration of a successful frame transmission and that of failed transmissions due to the erroneous data and ACK frames, respectively. Summing up all the time periods of the three scenarios $T_{a}, T_{b}$ and $T_{c}$ with the probabilities $p_{s}\left(l, s_{i}, M_{i}\right)$, $p_{e, \text { data }}\left(l, s_{i}, M_{i}\right)$, and $\left(1-p_{e, \text { data }}\left(l, s_{i}, M_{i}\right)\right) p_{e, a c k}\left(s_{i}\right)$, the average transmission time at the $i$ th transmission attempt $E\left[t_{T}\right]\left(l, s_{i}, M_{i}\right)$ can be expressed as

$$
\begin{aligned}
E\left[t_{T}\right] & \left(l, s_{i}, M_{i}\right) \\
= & p_{s}\left(l, s_{i}, M_{i}\right) \cdot T_{a}+p_{e, \text { data }}\left(l, s_{i}, M_{i}\right) \cdot T_{b} \\
& +\left(1-p_{e, \text { data }}\left(l, s_{i}, M_{i}\right)\right) p_{e, a c k}\left(s_{i}\right) \cdot T_{c} \\
& +\left(1-p_{s}(l, s, M)\right) \sum_{k=1}^{N} p_{j k} \cdot E\left[t_{T}\right]\left(l, s_{i+1}, M_{i+1}\right) .
\end{aligned}
$$

Denote $T_{\text {data }}$ and $T_{A C K}$ as the duration of data and ACK frame transmission, respectively. Based on Fig. 1, $T_{a}, T_{b}$, and 
$T_{c}$ can be written as follows:

$$
\left\{\begin{aligned}
T_{a}= & T_{\text {bkoff }}(i)+T_{\text {data }}\left(l, M_{i}\right) \\
& +T_{S I F S}+T_{A C K}+T_{D I F S} \\
T_{b}= & T_{\text {bkoff }}(i)+T_{\text {data }}\left(l, M_{i}\right)+T_{A C K \text { timeout }} \\
T_{c}= & T_{\text {bkoff }}(i)+T_{\text {data }}\left(l, M_{i}\right) \\
& +T_{S I F S}+T_{A C K}+T_{\text {EIFS }}
\end{aligned}\right.
$$

where the duration of an EIFS period $T_{E I F S}$, ACK timeout $T_{A C K t i m e o u t}$, and DIFS $T_{D I F S}$ specified in the IEEE Standard [16] are expressed as

$$
\begin{aligned}
T_{\text {EIFS }}= & T_{S I F S}+T_{A C K}+T_{D I F S}+T_{\text {preamble }} \\
& +T_{\text {PLCPhdr }} \\
T_{\text {ACKtimeout }}= & 2 \times T_{S I F S}+T_{A C K}+2 \times T_{\text {slot }} \\
T_{\text {DIFS }}= & T_{\text {SIFS }}+2 \times T_{\text {slot }} .
\end{aligned}
$$

Denote $C W_{\min }$ and $C W_{\max }$ as the minimum and maximum contention window sizes, respectively. Then, it follows that the average backoff time $T_{b k o f f}(i)$ at the $i$ th transmission attempt in (11) is equal to

$$
T_{b k o f f}(i)=\frac{\min \left[2^{i} \cdot\left(C W_{\min }+1\right)-1, C W_{\max }\right]}{2} \cdot T_{\text {slot }} .
$$

Moreover, given the bytes per symbol for the PHY mode $m$, as shown in Table I, $B p S(M), T_{d a t a}$, and $T_{A C K}$ are, respectively, written as

$$
\begin{aligned}
& T_{\text {data }}(l, M) \\
& =T_{\text {preamble }}+T_{\text {signal }}+T_{\text {sym }} \\
& \times\left\lceil\frac{l_{\text {data_MAChdr }}+l_{F C S}+\left(l_{\text {service }}+l_{\text {tail }}\right) / 8+l}{B p S(M)}\right\rceil \\
& =20 \mu s+T_{\text {sym }} \times\left\lceil\frac{30.75+l}{B p S(M)}\right\rceil \\
& T_{A C K} \\
& =T_{\text {preamble }}+T_{\text {signal }}+T_{\text {sym }} \\
& \times\left\lceil\frac{l_{A C K \_M A C h d r}+\left(l_{\text {service }}+l_{\text {tail }}\right) / 8}{B p S(1)}\right\rceil \\
& =20 \mu s+T_{\text {sym }} \times\left\lceil\frac{16.75}{B p S(1)}\right\rceil
\end{aligned}
$$

where $l_{M A C h d r}=24 \mathrm{~B}, l_{A C K}{ }_{M A C h d r}=14 \mathrm{~B}, l_{F C S}=4 \mathrm{~B}$, $l_{\text {service }}=16 \mathrm{~b}$, and $l_{\text {tail }}=6 \mathrm{~b}$ represent the length of the MAC header in data and ACK frames and the frame check sum field in the MAC header, service, and tail fields in the PLCP header, respectively. $T_{\text {preamble }}=16 \mu \mathrm{s}$ and $T_{\text {signal }}=4 \mu \mathrm{s}$ stand for the duration of the preamble and the signal field in the PLCDP header [16].

\section{B. Energy Efficiency}

Next, we derive the energy efficiency of the proposed CDRPA algorithm and the step-down retransmission strategy. Similar to the definition of the effective goodput, energy efficiency $\zeta$ is defined as the number of successfully delivered information bits per joule. In particular, $\zeta$ can be computed by the ratio of the average successfully delivered information bits of a data packet $E\left[P_{D}\right]$ to the total energy $E\left[\epsilon_{T}\right]$ that is required to transmit that packet, i.e.,

$$
\zeta\left(l, s_{i}, M_{i}\right)=\frac{E\left[l_{D}\right]\left(l, s_{i}, M_{i}\right)}{E\left[\epsilon_{T}\right]\left(l, s_{i}, M_{i}\right)} .
$$

Recall that $E\left[l_{D}\right]\left(l, s_{i}, M_{i}\right)$ can be obtained from (8) in the goodput computation.

Similar to the calculation of the average transmission time $E\left[t_{T}\right]\left(l, s_{i}, M_{i}\right)$ in (10), the total energy consumption for a data packet transmission $E\left[\epsilon_{T}\right]\left(l, s_{i}, M_{i}\right)$ also consists of the three scenarios in Fig. 1. Denote $\epsilon_{d a t a}\left(l, M_{i}, P_{t, i}\right)$ as the power consumption of a data frame at the $i$ th transmission attempt with the transmitted PHY mode $M_{i}$ and power level $P_{t, i}$. The energy consumption during the backoff time, ACK frame transmission, DIFS, and SIFS are given by $\epsilon_{b k o f f}, \epsilon_{A C K}$, $\epsilon_{D I F S}$, and $\epsilon_{S I F S}$, respectively. $E\left[\epsilon_{T}\right]\left(l, s_{i}, M_{i}\right)$ can be written by summing up all the energy consumption during the time periods of a successful packet transmission $T_{a}$ and the failed transmissions due to erroneous data and ACK frames $T_{b}$ and $T_{c}$, with the probabilities $p_{s}\left(l, s_{i}, M_{i}\right), p_{e, \text { data }}\left(l, s_{i}, M_{i}\right)$, and $\left(1-p_{e, \text { data }}\left(l, s_{i}, M_{i}\right)\right) p_{e, a c k}\left(s_{i}\right)$, i.e.,

$$
\begin{aligned}
E\left[\epsilon_{T}\right] & \left(l, s_{i}, M_{i}\right) \\
= & \epsilon_{\text {bkoff }}(i)+p_{s}\left(l, s_{i}, M_{i}\right) \\
& \cdot\left[\epsilon_{\text {data }}\left(l, M_{i}, P_{t, i}\right)+\epsilon_{S I F S}+\epsilon_{A C K}+\epsilon_{D I F S}\right] \\
& +p_{e, \text { data }}\left(l, s_{i}, M_{i}\right) \cdot\left[\epsilon_{\text {data }}\left(l, M_{i}, P_{t, i}\right)+\epsilon_{A C K t i m e o u t}\right] \\
& +\left(1-p_{e, \text { data }}(l, s, M)\right) \cdot p_{e, a c k}(s) \\
& \cdot\left[\epsilon_{\text {data }}\left(l, M, p_{t}\right)+\epsilon_{S I F S}+\epsilon_{A C K}+\epsilon_{E I F S}\right] \\
& +\left(1-p_{s}(l, s, M)\right) \cdot \sum_{k=1}^{N} p_{j k} \cdot E\left[\epsilon_{T}\right](l, r, M(r), c+1)
\end{aligned}
$$

Here, $\epsilon_{b k o f f}, \epsilon_{d a t a}, \epsilon_{A C K}, \epsilon_{D I F S}$, and $\epsilon_{S I F S}$ can, respectively, be expressed as

$$
\begin{aligned}
\epsilon_{b k o f f}(c) & =\overline{T_{b k o f f}}(c) \cdot P_{r \_m o d e} \\
\epsilon_{\text {data }}\left(l, M_{i}, P_{t, i}\right) & =T_{d a t a}\left(l, M_{i}\right) \cdot P_{t \_m o d e}\left(P_{t, i}\right) \\
\epsilon_{A C K} & =T_{A C K} \cdot P_{r \_m o d e} \\
\epsilon_{D I F S} & =T_{D I F S} \cdot P_{r \_m o d e} \\
\epsilon_{S I F S} & =T_{S I F S} \cdot P_{r \_m o d e}
\end{aligned}
$$

where the power consumption for a station that operates in the receiving $P_{r \_m o d e}$ and the transmitting modes $P_{t \_m o d e}\left(P_{t, i}\right)$ associated with transmitted power level $P_{t, i}$ at the $i \overline{\text { th }}$ transmission attempt can be expressed as follows:

$$
\left\{\begin{array}{l}
P_{r \_ \text {mode }}=P_{\text {com }}+P_{r e c} \\
P_{t \_m o d e}\left(P_{t, i}\right)=P_{c o m}+P_{p a}=P_{c o m}+\frac{P_{t, i}}{\eta\left(P_{t, i}\right)} .
\end{array}\right.
$$

Note that, in (25), $P_{r e c}$ and $P_{c o m}$ are the power consumption of the receiver front end and the shared components in both the receive and transmit circuits, respectively, $P_{p a}$ is the power consumption of the power amplifier in the transmit circuit, and $\eta$ is the power conversion efficiency of the power amplifier. 
TABLE $\mathrm{V}$

SYSTEM PARAMETERS IN NUMERICAL RESULTS

\begin{tabular}{|c|c|}
\hline Parameters & Value \\
\hline \hline packet payload & 1500 bytes \\
\hline ACK & 112 bits \\
\hline PLCP preamble duration & $16 \mu \mathrm{s}$ \\
\hline PLCP signal field duration & $4 \mu \mathrm{s}$ \\
\hline OFDM symbol time & $4 \mu \mathrm{s}$ \\
\hline Slot time & $9 \mu \mathrm{s}$ \\
\hline SIFS & $16 \mu \mathrm{s}$ \\
\hline DIFS & $34 \mu \mathrm{s}$ \\
\hline$C W_{\min }$ & 15 \\
\hline$C W_{\max }$ & 1023 \\
\hline$P_{t(\max )}$ & $30 \mathrm{dBm}$ \\
\hline
\end{tabular}

\section{NumericAl RESUlts}

In this section, we compare the energy efficiency and goodput performance of the proposed rate-first and power-first CDRPA algorithms and the energy-optimal complete searching method [3]. Note that the complete searching method exhaustively searches among all the transmitted rates and power levels to maximize the energy efficiency. The related system parameters are summarized in Table V.

\section{A. Energy Efficiency Performance}

Fig. 4(a) and (b) shows the energy efficiency for the three algorithms in the AWGN and Rayleigh fading channels, respectively. As shown in the figure, the energy efficiency of all the three algorithms in the AWGN channel is better than in the Rayleigh fading channel. The signal strength varies with time in the Rayleigh fading channel; therefore, the channelstate information from the previously received ACK frame may not appropriately reflect the current channel condition. This phenomenon makes the station use the improper rate and power level and, thus, decreases the energy efficiency in the Rayleigh channel due to the increased frame retransmissions.

In addition, one can observe that the power-first CDRPA scheme has about the same energy efficiency performance as the energy-optimal complete search algorithm, particularly in the Rayleigh fading channel. In the AWGN channel, the power-first CDRPA scheme has slightly lower efficiency than the complete-search approach at certain $E_{b} / N_{0}$ values due to the lack of an appropriate PHY mode. Both link adaptation algorithms provide better energy efficiency than the rate-first CDRPA scheme.

Another interesting point is that the energy efficiency of the rate-first CDRPA scheme experiences a sudden drop at the $E_{b} / N_{0}$ thresholds of different PHY modes. When the channel quality is good, the rate-first CDRPA scheme chooses a faster transmitted rate instead of reducing the transmitted power. A higher PHY mode needs to use a higher transmitted power level to satisfy the PER requirement; therefore, the energy efficiency of the rate-first CDRPA scheme experiences a sudden drop at the $E_{b} / N_{0}$ thresholds.

\section{B. Goodput Performance}

Fig. 5(a) and (b) shows the goodput performance of the three algorithms in the AWGN and Rayleigh fading channels,

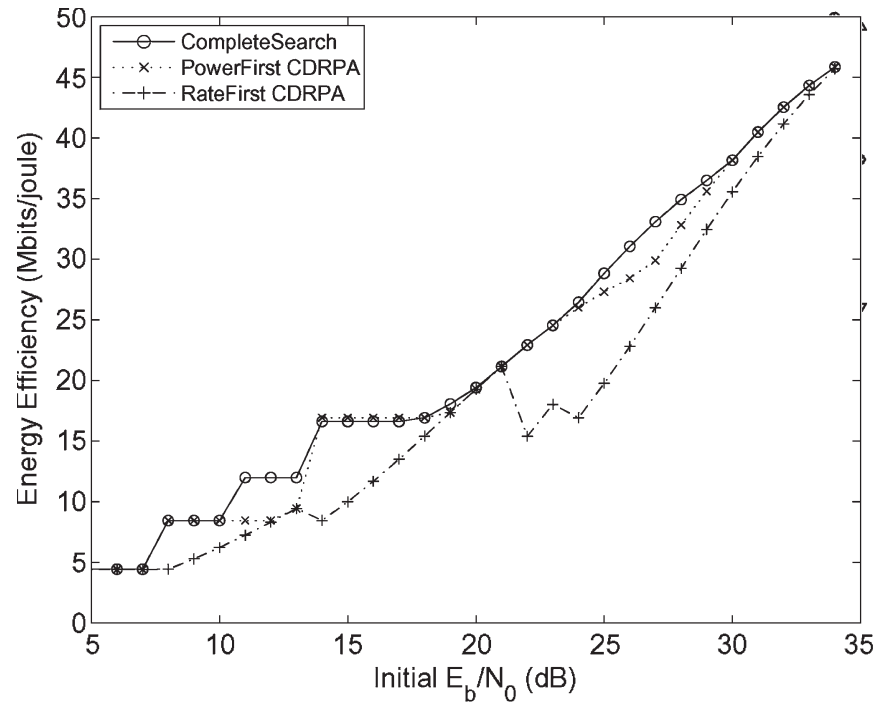

(a)

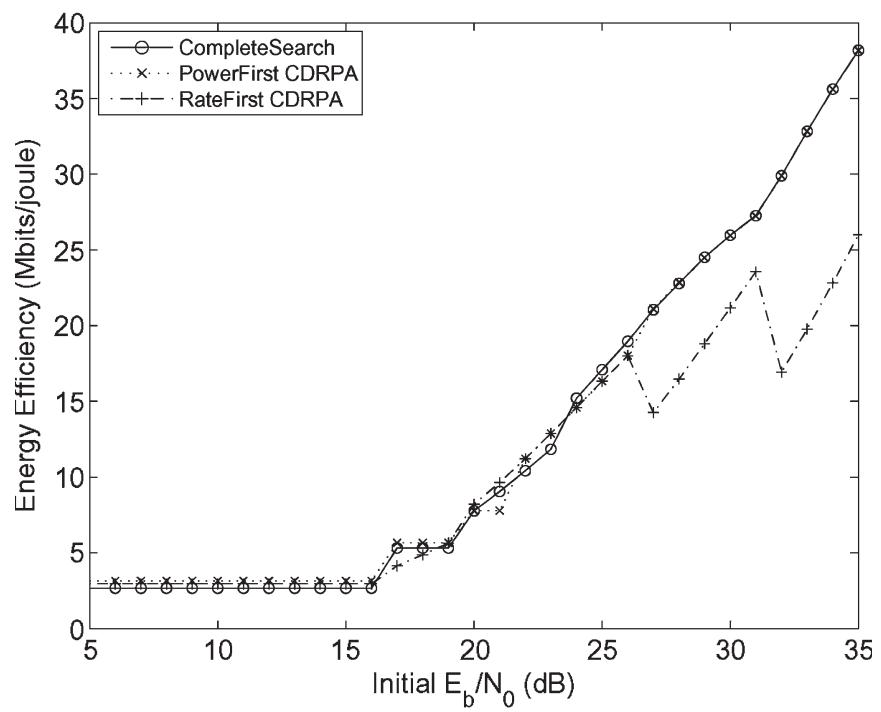

(b)

Fig. 4. Comparison of energy efficiency performance in (a) the AWGN channel and (b) the Nakagami fading channel, with $m=1$, respectively.

respectively. As shown in the figure, the rate-first CDRPA scheme provides the highest goodput of at most $E_{b} / N_{0}$ values compared with the other two algorithms. Unlike the other two algorithms, which reduce the transmitted power level, the ratefirst CDRPA schemes choose higher transmitted rates when the channel quality improves. Thus, the latter scheme has better goodput performance. For $E_{b} / N_{0}=30 \mathrm{~dB}$, the goodput performance of the rate-first CDRPA scheme in the Rayleigh fading channel is larger than the other schemes by almost $50 \%$.

However, for $E_{b} / N_{0}=8 \sim 18 \mathrm{~dB}$ in the AWGN channel, the rate-first CDRPA algorithm has lower goodput performance than the power-first CDRPA and the complete search schemes. In this situation, the two energy-optimized schemes adopt a faster rate to save the transmission time and, thus, the energy consumption. Although a higher PHY mode leads to a higher packet error probability, the step-down retransmission strategy quickly recovers the transmission without increasing too many retransmissions. On the other hand, the rate-first CDRPA only 


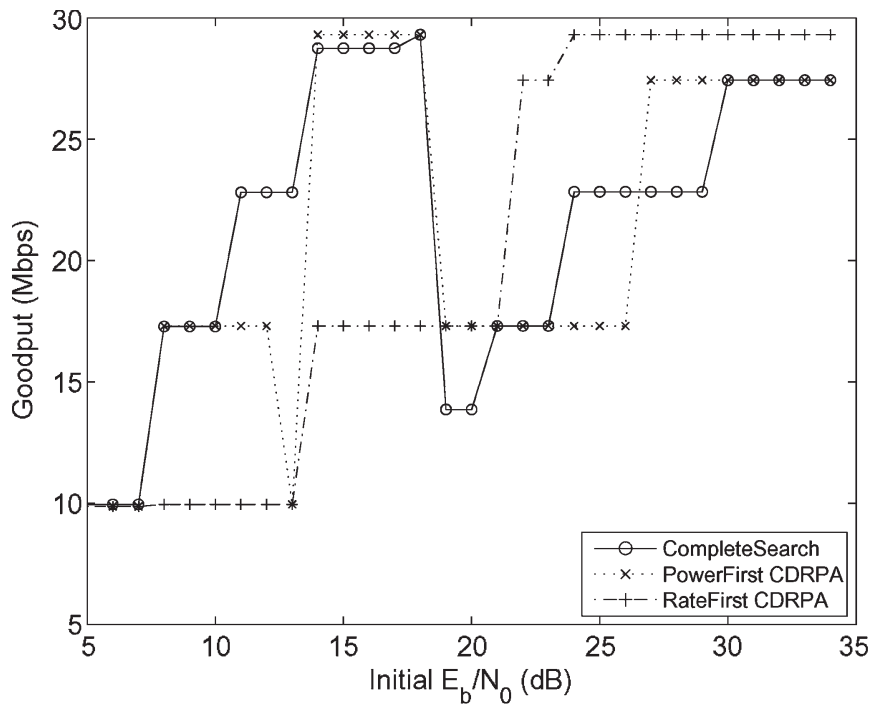

(a)

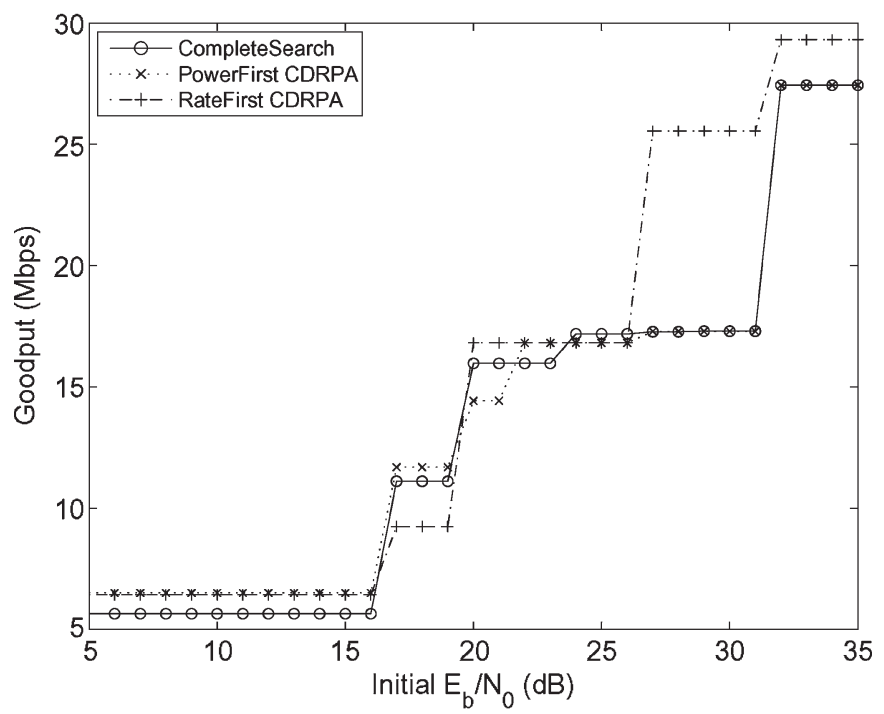

(b)

Fig. 5. Comparison of goodput performance in (a) the AWGN channel and (b) the Nakagami fading channel, with $m=1$, respectively.

uses the PHY mode that can satisfy the PER requirement. Thus, the goodput performance for the rate-first CDRPA scheme is not always the highest due to the use of lower but sustainable PHY modes.

\section{Impact of the Nakagami Shape Factor}

Next, we investigate the impact of the Nakagami shape factor $m$ on the energy efficiency and goodput performance of the power-first and rate-first CDRPA algorithms. As shown in Fig. 6(a) and (b), the smaller the Nakagami factor $m$, the lower the energy efficiency and the goodput performance. This observation results from the fact that all the PHY modes in the Nakagami fading channel with a smaller factor $m$ have larger $E_{b} / N_{0}$ thresholds. The station only uses a slower transmitted rate and a higher transmitted power level for the frame transmission, given an $E_{b} / N_{0}$ value. Therefore, both the energy efficiency and goodput performance in the channel with a small Nakagami factor $m$ are decreased.

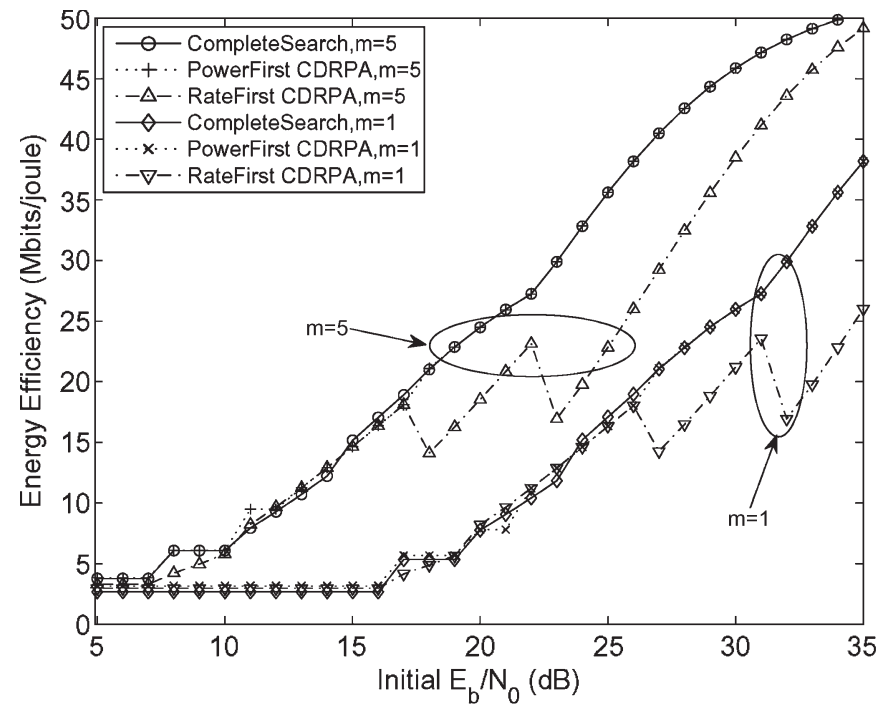

(a)

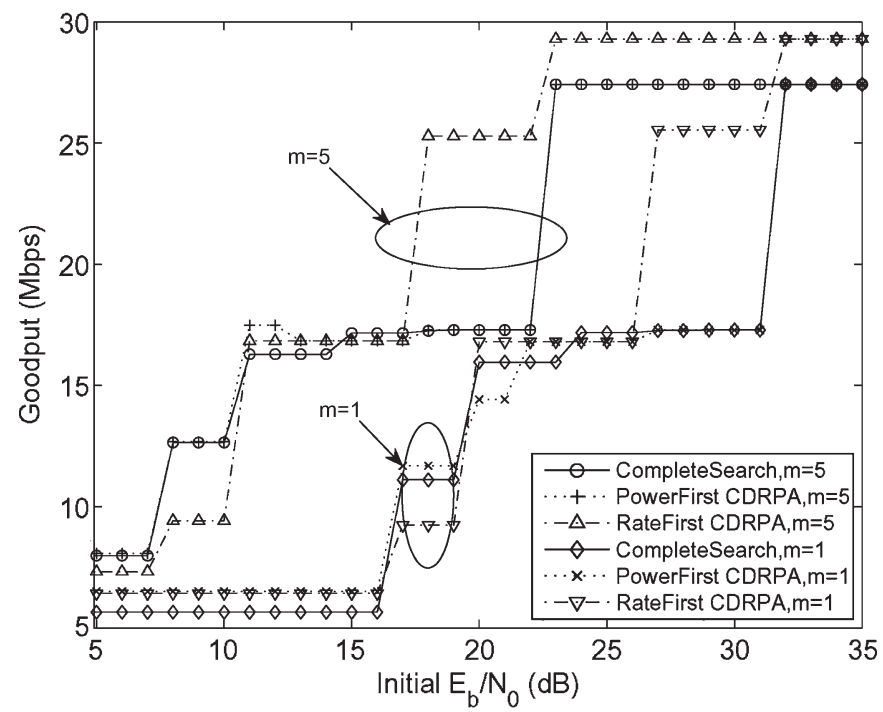

(b)

Fig. 6. Impact of the Nakagami shaping factor on (a) energy efficiency and (b) goodput for different joint rate and power adaptation schemes.

Surprisingly, in the Rayleigh fading channel and the cases of low-SNR region (i.e. $E_{b} / N_{0}<20 \mathrm{~dB}$ ), the power-first CDRPA algorithm outperforms the complete search scheme for both the energy efficiency and goodput performance due to the use of the efficient and fast PHY mode. On the other hand, as the channel has a stronger line-of-sight component, e.g., the AWGN or the Nakagami channel with $m=5$, both the power-first algorithm and the complete search scheme have similar energy efficiency and goodput performance. Therefore, we claim that the proposed power-first algorithm, using the reduced table, is more appropriate than the complete search scheme in the Rayleigh and Nakagami fading channels with smaller values of $m$.

\section{Effective Power Consumption}

As shown in Fig. 6, the power-first CDRPA algorithm outperforms the rate-first CDRPA algorithm in terms of energy efficiency at the cost of sacrificing the goodput performance. To explicitly compare the performance of the two adaptation 


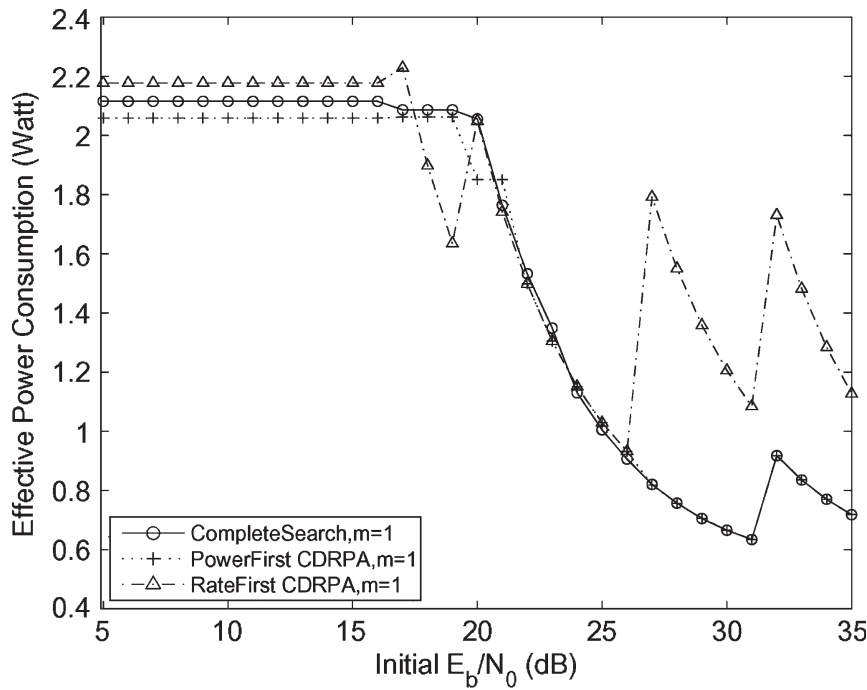

(a)

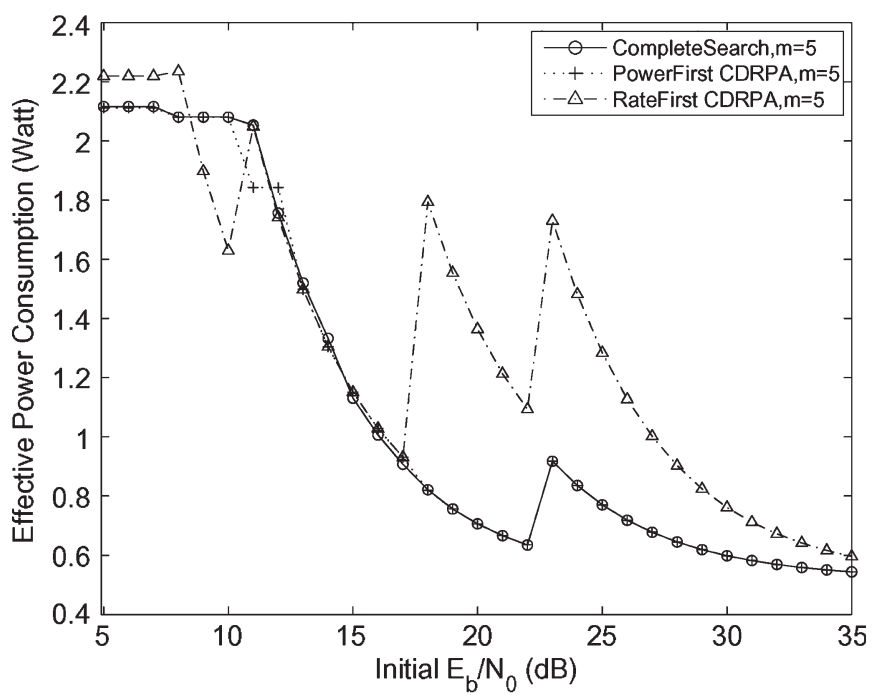

(b)

Fig. 7. Effective power consumption of the complete search, power-first CDRPA, and rate-first CDRPA algorithms in the Nakagami fading channel with shape factors (a) $m=1$ and (b) $m=5$.

algorithms in terms of the tradeoff between the energy efficiency and goodput performance, a new performance metric, i.e., effective power consumption $P_{c}$, is defined as the ratio of goodput $G$ to energy efficiency $(\zeta)$, i.e., $P_{c}=G / \zeta$. According to this definition, this metric is given in watts; thus, $P_{c}$ can be considered as the required power consumption when a station has successfully delivered an information bit. As shown in Fig. 7, the power-first CDRPA scheme has lower effective power consumption for most cases of initial $E_{b} / N_{0}$ values. Therefore, we conclude that the power-first CDRPA scheme is better than the rate-first version in terms of the tradeoff between the energy efficiency and goodput performance.

\section{E. Impact of Time Correlation in the Nakagami Fading Channel}

Fig. 8(a) and (b) demonstrates the impact of the time correlation values $\tau$ of (4) in the Nakagami fading channels on

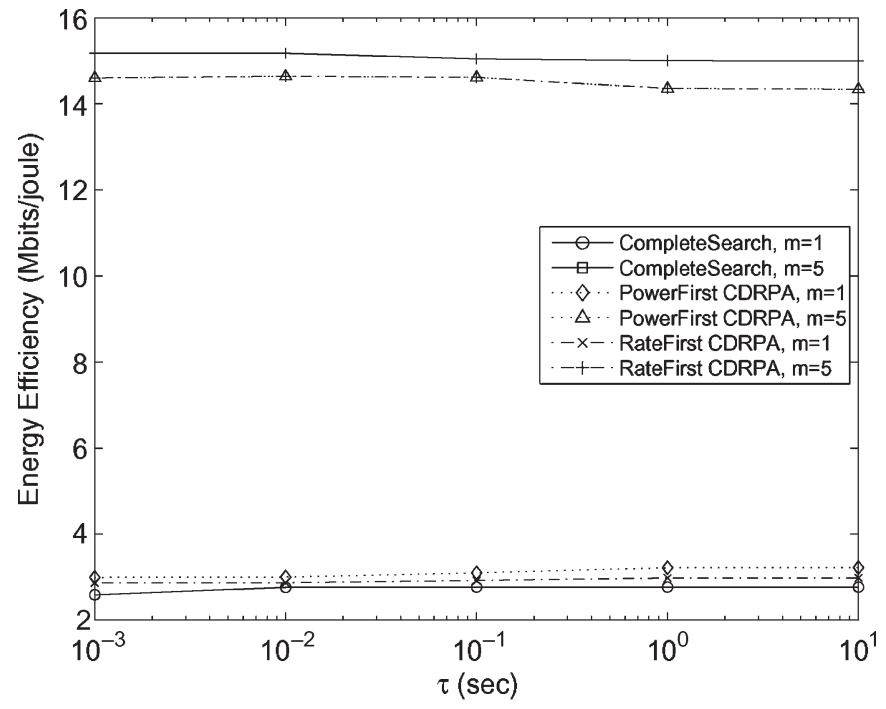

(a)

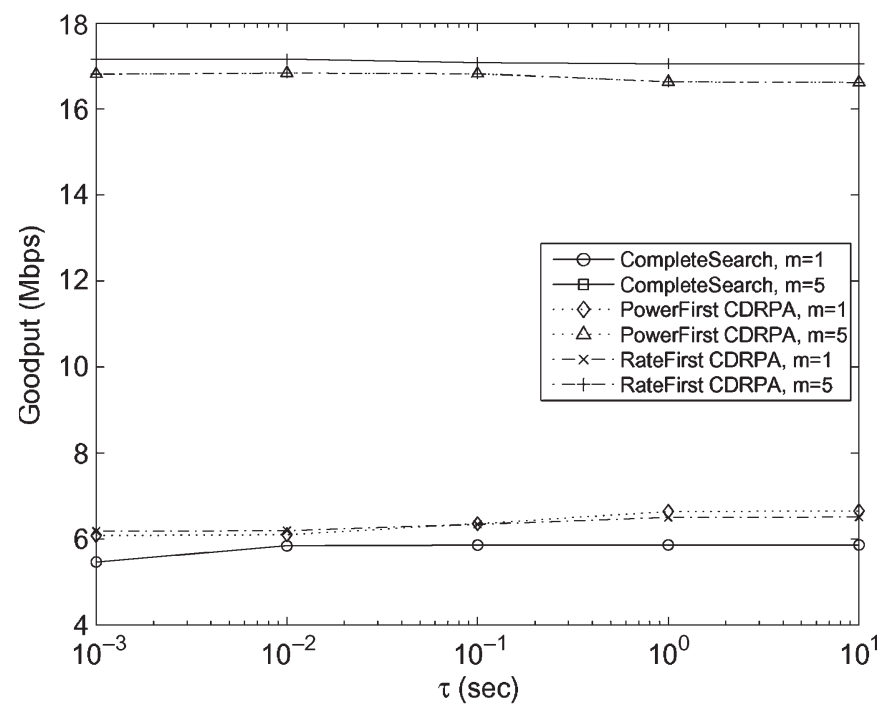

(b)

Fig. 8. Impact of time correlation on (a) energy efficiency and (b) goodput performance in the Nakagami fading channel, with $m=1$ and $m=5$, at $E_{b} / N_{0}=14 \mathrm{~dB}$, respectively.

the energy efficiency and goodput performance of the powerfirst and rate-first CDRPA schemes. As shown in the figure, the values of $\tau$ have insignificant impact on both energy efficiency and goodput performance. For example, for the case of the power-first CDRPA scheme in the Nakagami fading channel, with $m=1$, the energy efficiency holds at $3.1 \mathrm{Mb} / \mathrm{J}$ and goodput performance $6.5 \mathrm{Mb} / \mathrm{s}$ when $\tau=10^{-3} \sim 10 \mathrm{~s}$. This phenomenon results from the design of the step-down retransmission strategy, in which the station adjusts the transmitted rate and power level, depending on the previous selection at the first transmission attempt instead of the current channel-state information. Thus, the changes in $E_{b} / N_{0}$ have an insignificant impact on both the energy efficiency and goodput performance.

Fig. 9(a) and (b) shows the impact of time correlation values $\tau$ in the Nakagami fading channels on the energy efficiency and goodput performance of the power-first and rate-first CDRPA schemes with the beamwidth parameter $\kappa=2$. The parameter $\kappa$ controls the beamwidth of nonisotropic and time-correlated 


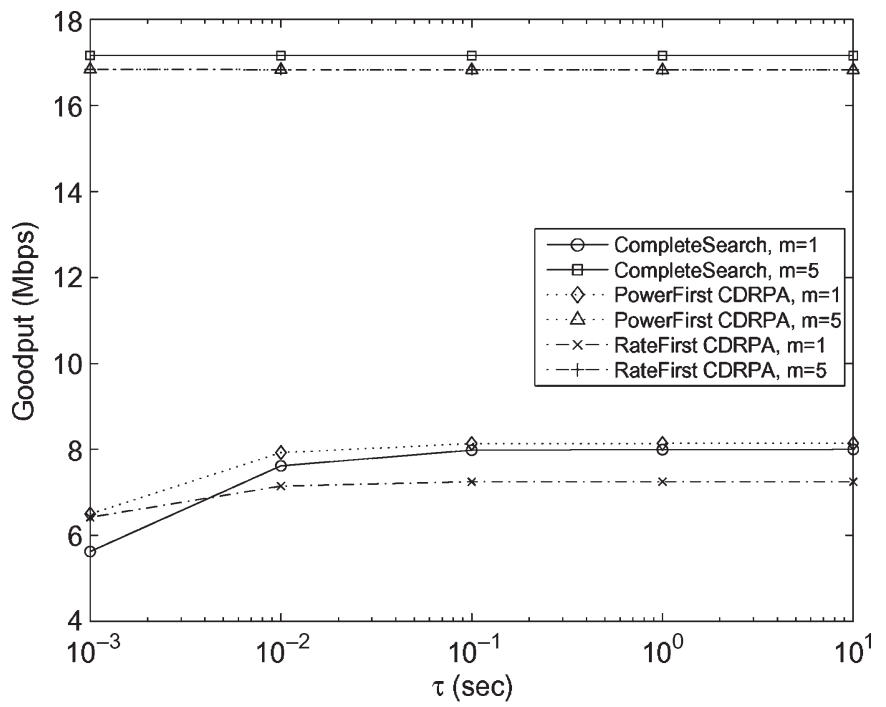

(a)

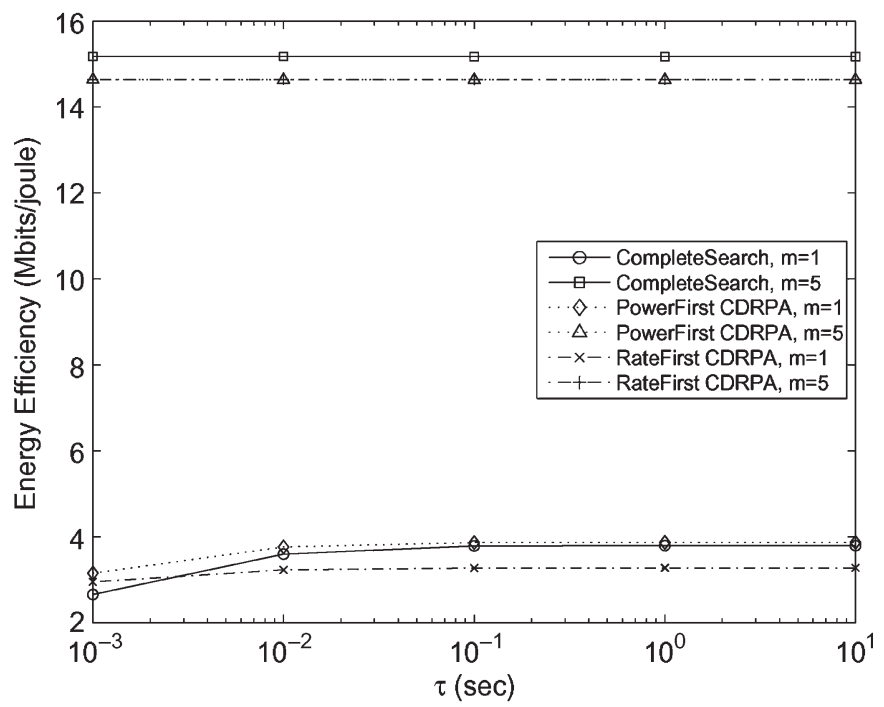

(b)

Fig. 9. Impact of time correlation on (a) energy efficiency and (b) goodput performance in the Nakagami fading channel, with $m=1$ and $m=5$, at $E_{b} / N_{0}=14 \mathrm{~dB}$, respectively. The beamwidth parameter $\kappa=2$.

Nakagami- $m$ fading scenarios [18]. In Fig. 8, the parameter $\kappa=0$. As shown in Fig. 9, the values of $\tau$ have an insignificant impact on both energy efficiency and goodput performance for $m=5$. However, when $m=1$, the energy efficiency and goodput increase as $\tau$ increases.

\section{F. Comparison on Computation Complexity}

Table VI compares the number of combinations for rate and power adaptation between the complete-search method and the power-first CDRPA algorithm. According to the IEEE $802.11 \mathrm{a} / \mathrm{g}$ Standard, eight PHY modes are provided for the transmitted rate selection. Given 15 levels for the transmitted power adaptation, the complete-search method requires searching all the combinations among the supported rates and power levels (i.e., $8 \times 15=120$ options). Note that the larger the power level that a station can use, the larger the combination that the complete-search method has to search. On the other
TABLE VI

COMPUTATIONAL COMPLEXITY COMPARISON

\begin{tabular}{|c|c|c|c|}
\hline $\begin{array}{c}\text { Number of } \\
\text { Computation }\end{array}$ & Complete Search & CDRPA & $\begin{array}{c}\text { Complexity } \\
\text { Reduction }\end{array}$ \\
\hline \hline AWGN $(m=\infty)$ & $8 \times 15$ & 7 & $94.16 \%$ \\
\hline Nakagami $(m=5)$ & $8 \times 15$ & 5 & $95.83 \%$ \\
\hline Rayleigh $(m=1)$ & $8 \times 15$ & 4 & $96.67 \%$ \\
\hline
\end{tabular}

hand, the power-first CDRPA scheme first determines the transmitted power levels for the supported rates in the reduced-mode table based on the available link margin and then selects the transmitted rate with the maximum energy efficiency for the next frame transmission. Therefore, the number of comparisons for the power-first CDRPA scheme is equal to the size of the reduced-mode table. Therefore, the computation complexity of the power-first CDRPA algorithm in the AWGN and Nakagami fading channels can be reduced by $94 \% \sim 97 \%$, respectively. The complete search for 120 different options does not seem unfeasible for current processing capabilities. Nevertheless, the dynamic range of power and the options of rates may be huge in future wireless systems. In that case, the complexity reduction that is offered by the suggested joint rate and power-adaptation scheme can result in significant advantages.

\section{CONCLUSION}

In this paper, we have presented the rate-first and power-first CDRPA algorithms for WLANs in Nakagami fading channels. Both adaptation schemes quickly respond to the fast varying wireless channel in determining the transmitted rate and power with low computational complexity. We have also designed the step-down retransmission strategy for the case where the ACK frame is not successfully received at the source station. The designed strategy only relies on the selection at the first transmission attempt without requiring channel-state information. In addition, a PHY/MAC cross-layer analytical model was developed to evaluate the goodput and energy efficiency for the WLAN in generalized Nakagami channels. The powerfirst CDRPA mechanism can not only deliver almost the same energy efficiency as the energy-optimal link adaptation scheme but can also have comparable goodput to the goodput-optimal link adaptation mechanism in the Rayleigh fading channel. As for the computational complexity, the CDRPA algorithm can reduce $94 \%$ of the comparisons than the complete search mechanisms. Hence, considering the overall tradeoff among energy efficiency, goodput, and computational complexity, the power-first CDRPA mechanism is an appropriate option in determining the transmitted power levels and rates compared with other mechanisms in the literature.

\section{REFERENCES}

[1] Part 11: Wireless LAN Medium Access Control (MAC) and Physical Layer (PHY) Specifications: Spectrum and Transmit Power Management extensions in the 5- GHz band in Europe, IEEE Std. 802.11h, Oct. 2003.

[2] D. Qiao, S. Choi, and K. G. Shin, "Goodput analysis and link adaptation for IEEE 802.11a wireless LANs," IEEE Trans. Mobile Comput., vol. 1, no. 4, pp. 278-292, Oct.-Dec. 2002. 
[3] D. Qiao, S. Choi, A. Jain, and K. G. Shin, "MiSer: An optimal low-energy transmission strategy for IEEE 802.11a/h," in Proc. ACM Annu. Int. Conf. Mobile Comput. Netw., Sep. 2003, pp. 14-19.

[4] E. S. Jung and N. Vaidya, "An energy efficient MAC protocol for wireless LANs," in Proc. IEEE INFOCOM, Jun. 23-27, 2002, vol. 3, pp. 1756-1764.

[5] P. G. T. Simunic, L. Benini, and G. D. Micheli, "Dynamic power management for portable systems," in Proc. ACM Annu. Int. Conf. Mobile Comput. Netw., Aug. 2000, pp. 11-19.

[6] S. Choi and A. A. Soomro, "Updating path loss estimation for power control and link adaptation in IEEE 802.11h WLAN," U.S. Patent 6978 151, Dec. 20, 2005.

[7] A. Kamerman and L. Monteban, "WaveLAN-II: A high-performance wireless LAN for the unlicensed band," Bell Syst. Tech. J., vol. 2, no. 3, pp. 118-133, Summer 1997.

[8] M. Lacage, M. H. Manshaei, and T. Turletti, "IEEE 802.11 rate adaptation: A practical approach," in Proc. 7th ACM Int. Symp. Model., Anal. Simul. Wireless Mobile Syst., Oct. 2004, pp. 126-134.

[9] U. Eckhardt, M. Lenk, and Grell, "Transmitter adjustment based transmission statistics," U.S. Patent 7418 054, Aug. 26, 2008.

[10] J. D. Prado and S. Choi, "Method and system for generating and updating transmission rate for link adaptation in IEEE 802.11 WLAN," U.S. Patent 7336 634, Feb. 26, 2008.

[11] G. Holland, N. Vaidya, and P. Bahl, "A rate-adaptive MAC protocol for multihop wireless networks," in Proc. 7th Annu. Int. Conf. Mobile Comput. Netw., Jul. 2001, pp. 236-251.

[12] L. C. Wang, Y. W. Lin, and W. C. Liu, "Cross-layer goodput analysis for rate adaptive IEEE 802.11a WLAN in the generalized Nakagami fading channel," in Proc. IEEE Int. Conf. Commun., Jun. 2004, vol. 4, pp. 2312-2316.

[13] W. C. Liu, L. C. Wang, and Y. W. Lin, "Physical layer effects on the MAC goodput performance for the rate adaptive IEEE 802.11a/g WLAN," in Proc. IEEE Wireless Commun. Netw. Conf., Mar. 21-25, 2004, vol. 3, pp. 1873-1878.

[14] Part 11: Wireless LAN Medium Access Control (MAC) and Physical Layer (PHY) Specifications: High-Speed Physical Layer in the $5 \mathrm{GHz}$ Band, IEEE Std. 802.11a, Sep. 1999. supplement to IEEE 802.11 Standard.

[15] B. O'Hara and A. Petrick, The IEEE 802.11 Handbook: A Designer's Companion. New York: IEEE, 1999.

[16] Part 11: Wireless LAN Medium Access Control (MAC) and Physical Layer (PHY) Specifications, IEEE Std. 802.11, Aug. 1997. Standard, IEEE.

[17] M. Nakagami, Statistical Methods in Radio Wave Propagation. Oxford, U.K.: Pergamon, 1960.

[18] J. C. S. S. Filho, M. D. Yacoub, and G. Fraidenraich, "A simple accurate method for generating autocorrelated Nakagami-m envelope sequences," IEEE Commun. Lett., vol. 11, no. 3, pp. 231-233, Mar. 2007.

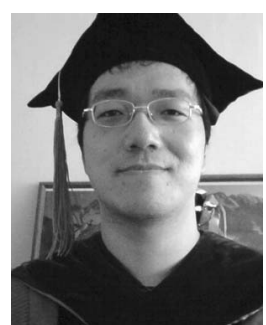

Wei-Cheng Liu (S'04-M'08) received the B.S. and M.S. degrees in electrical engineering from the National Tsing Hua University, Hsinchu, Taiwan, in 1999 and 2001. He is currently working toward the Ph.D. degree with the Department of Communication Engineering, National Chiao Tung University, Hsinchu.

He served the military service in Cheng Gong Ling, Taichung, Taiwan. In 2002, he was a GSM Layer-1 Software Engineer with Compal Communications, Taipei, Taiwan. His research interests include MIMO Rician channels in mobile ad hoc networks, cross-layer rate and power adaptation for wireless LANs, performance analysis for UWB systems, space-time-frequency code design, and cooperative network coding.

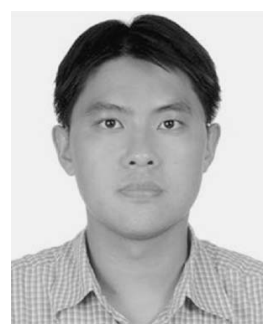

Anderson Chen (S'04-M'08) received the B.S. M.S., and Ph.D. degrees in communication engineering from the National Chiao Tung University, Hsinchu, Taiwan, in 1998, 1999, and 2007, respectively.

He is currently working on his national service. His research interests include wireless networks, cross-layer design, and cognitive wireless systems. $\mathrm{He}$ is the holder of one U.S. patent and has three patents pending.

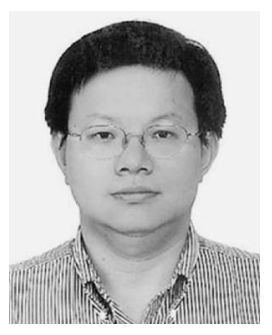

Li-Chun Wang (S'92-M'96-SM'06) received the B.S. degree in electrical engineering from the $\mathrm{Na}-$ tional Chiao Tung University, Hsinchu, Taiwan, in 1986, the M.S. degree in electrical engineering from the National Taiwan University, Taipei, Taiwan, in 1988, and the M.Sc. and Ph.D. degrees in electrical engineering from Georgia Institute of Technology, Atlanta, in 1995 and 1996, respectively.

From 1990 to 1992, he was with Chunghwa Telecom, Taoyuan, Taiwan. In 1995, he was with Northern Telecom, Richardson, TX. From 1996 to 2000, he was a Senior Technical Staff Member with the Wireless Communications Research Department, AT\&T Laboratories. In August 2000, he became an Associate Professor with the Department of Communication Engineering, National Chiao Tung University, where he has been a Full Professor since August 2005. His research interests include cellular architectures, radio network resource management, and cross-layer optimization for cooperative and cognitive wireless networks. He is the holder of three U.S. patents and has three patents pending.

Dr. Wang is a corecipient of the Jack Neubauer Best Paper Award from the IEEE Vehicular Technology Society in 1997.

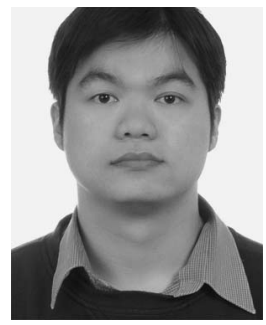

Kuang-Nan Yen was born in Taiwan in 1981. He received the B.S. degree in electrical engineering from I-Shou University, Kaohsiung, Taiwan, in 2003 and the M.S. degree from the National Chiao Tung University, Hsinchu, Taiwan.

$\mathrm{He}$ is currently with TrendChip Technologies Corp., Hsinchu. His research interests include wireless communications. 